\title{
High glucose-induced oxidative stress and mitochondrial dysfunction in neurons
}

\author{
JAMES W. RUSSELL, ${ }^{*},{ }^{+1}$ DAVID GOLOVOY,${ }^{*}{ }^{\dagger}$ ANDREA M. VINGENT,* PIA MAHENDRU,* \\ JAMES A. OLZMANN,* ALICE MENTZER, $* \dagger$ AND EVA L. FELDMAN* \\ *Department of Neurology, University of Michigan, Ann Arbor, Michigan, USA; and ${ }^{\dagger}$ Department of \\ Neurology, Veterans Affairs Medical Center, Ann Arbor, Michigan, USA
}

\begin{abstract}
The current study examines the association between glucose induction of reactive oxygen species (ROS), mitochondrial (Mt) depolarization, and programmed cell death in primary neurons. In primary dorsal root ganglion (DRG) neurons, $45 \mathrm{mM}$ glucose rapidly induces a peak rise in ROS corresponding to a $50 \%$ increase in mean Mt size at $6 \mathrm{~h}(P<0.001)$. This is coupled with loss of regulation of the Mt membrane potential (Mt membrane hyperpolarization, followed by depolarization, MMD), partial depletion of ATP, and activation of caspase-3 and -9. Glucose-induced activation of ROS, MMD, and caspase- 3 and -9 activation is inhibited by myxothiazole and thenoyltrifluoroacetone $(P<0.001)$, which inhibit specific components of the Mt electron transfer chain. Similarly, MMD and caspase-3 activation are inhibited by $100 \mu \mathrm{M}$ bongkrekic acid (an inhibitor of the adenosine nucleotide translocase ANT). These results indicate that mild increases in glucose induce ROS and Mt swelling that precedes neuronal apoptosis. Glucotoxicity is blocked by inhibiting ROS induction, MMD, or caspase cleavage by specific inhibitors of electron transfer, or by stabilizing the ANT.-Russell, J. W., Golovoy, D., Vincent, A. M., Mahendru, P., Olzmann, J. A., Mentzer, A., Feldman, E. L. High glucose-induced oxidative stress and mitochondrial dysfunction in neurons. FASEBJ. 16, 1738-1748 (2002)
\end{abstract}

Key Words: apoptosis $\cdot$ mitochondria $\cdot D R G \cdot$ glucose $\cdot$ reactive oxygen species

INCREASING EVIDENCE INDICATES that mitochondria are intimately associated with programmed cell death (PCD) in neurons (for review, see ref 1 ). The mechanism(s) leading to mitochondrial (Mt) dysfunction and apoptosis in neurons is uncertain; however, oxidative stress may be central to depolarization of the inner $\mathrm{Mt}$ membrane potential (loss of the $\Delta \Psi_{\mathrm{M} \text { ) }}$, and may initiate PCD. This association between oxidative stress and PCD is important in the pathogenesis of several neurodegenerative diseases, such as Alzheimer's disease, Huntington's disease, the muscular dystrophies, and in models of cerebral ischemia. Loss of the $\Delta \Psi_{\mathrm{M}}$ leads to inner Mt membrane depolarization (MMD) and release of cytochrome $c$ and other regulatory proteins from the Mt (1). Release of cytochrome $c$ into the cytosol activates apoptosis-activating factor I (Apaf-1) and caspase-9, with formation of a cytochrome $c /$ caspase-9/Apaf-1 complex (the apoptosome) (1).

Previous studies in this and other laboratories show that high glucose induces PCD in neurons both in vivo and in vitro (2-6). In primary dorsal root ganglion neurons (DRG) from diabetic rats, there is downregulation of Bcl-2 (3) and evidence of apoptosis $(2,3)$. Several laboratories have shown in diabetic rats a loss of DRG neurons, particularly large neurons, corresponding to loss of the largest myelinated fibers, resulting in neuropathy $(2,7,8)$. Diabetic neuropathy in animals is associated with induction of a variety of reactive oxygen species (ROS) and may be ameliorated by antioxidant treatment (9). Although a clear link between oxidative stress and neuronal PCD is established for acute and chronic neurodegenerative diseases, the mechanism(s) by which high glucose induces neuronal PCD in animal and culture models of diabetic neuropathy is unknown. This study explores how oxidative stress and changes in the $\Delta \Psi_{\mathrm{M}}$ induce PCD in primary neurons. DRG cultured in high glucose accumulate ROS, followed by Mt swelling, initial Mt hyperpolarization, then depolarization and efflux of cytochrome $c$ into the cytosol. Cytochrome $c$ efflux activates caspase-3 and -9 resulting in DRG apoptosis. These changes are blocked by inhibitors of the Mt respiratory chain and stabilization of the $\Delta \Psi_{\mathrm{M}}$, indicating that glucose activates the caspase cascade in neurons by oxidative stress-induced Mt dysfunction.

\section{MATERIALS AND METHODS}

Mitotracker orange (CMTMRos), chloromethyl-dichlorodihydrofluorescein diacetate (CM- $\mathrm{H}_{2}$ DCFDA), tetramethylrhodamine ester (TMRM), and JC1 were obtained from Molecular Probes, Eugene, OR. For ADP:ATP measurements, a luciferase kit was obtained from Lumitech Ltd. (Nottingham, UK). Bongkrekic acid (BKA) was purchased from Biomol (Plymouth Meeting, PA), Boc-aspartyl (OMe)-fluoromethylketone (BAF) from Enzyme Systems Products (Dublin, CA), and neurobasal medium, B27, and trypsin from Life Technol-

\footnotetext{
${ }^{1}$ Correspondence University of Michigan, Department of Neurology, 200 Zina Pitcher Place, 4410 Kresge III, Ann Arbor, MI 48109-0588, USA. E-mail: jruss@umich.edu
} 
ogies (Grand Island, NY). The CM1 antibody was a gift from Dr. A. Srinivasan of IDUN Pharm., La Jolla, CA. CaspaTag anti-active caspase-9 was from Intergen (Purchase, NY); cytochrome $c$ MAb (clone 6H2.B4 PharMingen) and secondary antibodies were from Vector (Burlingame, CA). All other chemicals were purchased from Sigma Chemical Co. (St. Louis, MO).

\section{DRG culture}

Dissociated DRG were prepared and aseptically plated on air-dried, collagen-coated plastic ACLAR dishes or plastic 12-well plates as described (2). To reduce the number of contaminating Schwann cells and fibroblasts by $>95 \%$, neurons were treated with $30 \mu \mathrm{M}$ 5-fluoro-2'-deoxyuridine. Dissociated DRG neurons were plated at density of $\sim 5000$ neurons per ACLAR dish. DRG were incubated at $37^{\circ} \mathrm{C}$ with $5 \%$ carbon dioxide; initial plating and culture was in serumfree B27 without antioxidants and neurobasal medium. For all experiments, cultures were washed and cultured in a defined medium containing neurobasal medium supplemented with $1.4 \mathrm{mM}$ L-glutamine, $30 \mathrm{nM}$ selenium, $10 \mathrm{nM}$ hydrocortisone, $10 \mathrm{nM} \beta$-estriadiol, and $10 \mu \mathrm{g} / \mathrm{mL}$ transferrin. We earlier reported that serum and insulin-free media produce equal or superior neuronal growth and survival $(0-96 \mathrm{~h})$ as media containing calf serum (2). Optimal DRG survival and neurite growth require $25-30 \mathrm{mM}$ basal glucose (2), reflecting the fact that neurons have high metabolic rates. Neurobasal media containing $25 \mathrm{mM}$ glucose meets these metabolic requirements. This concentration is subsequently called control glucose. A high glucose condition contains at least $20 \mathrm{mM}$ added glucose (total of $45 \mathrm{mM}$ glucose). DRG were cultured in control or high glucose medium $\pm 100 \mu \mathrm{M}$ BKA or $3 \mu \mathrm{M}$ myxothiazole for $0-24 \mathrm{~h}$. These concentrations of BKA and myxothiazole have been shown to have maximum efficacy in DRG neurons over 6-24 $\mathrm{h}$ without inducing PCD.

\section{Measurement of ROS}

Cell-permeant CM- $\mathrm{H}_{2}$ DCFDA was dissolved in DMSO as a concentrate and diluted to $0.1 \%$ DMSO. This concentration of DMSO is nontoxic to DRG cultures, does not affect measurements of oxidative stress or Mt dysfunction, and is unlikely to act as a hydroxyl ion trap. In the presence of ROS, intracellular esterases cleave acetate groups from CM$\mathrm{H}_{2}$ DCFDA, trapping nonfluorescent 2', 7'-dichlorofluorescein in the neuron (10). Subsequent oxidation yields fluorescent DCF, which detects transient rises in ROS, including $\mathrm{H}_{2} \mathrm{O}_{2}$ and, in particular, peroxinitrites (10). To confirm that DCF was able to detect increased oxidative stress within this culture system, DRG in control medium were treated with 4 $\mu \mathrm{M}$ antimycin A. Using confocal microscopy, DCF levels peaked with antimycin A at $1 \mathrm{~h}$ compared with control. DCF similarly showed a dose-dependent increase in DRG neurons exposed to $\mathrm{H}_{2} \mathrm{O}_{2}$. Having established that DCF accurately and sensitively measures ROS generation in cultured neurons, DRG were coincubated with $2 \mu \mathrm{M}$ CM- ${ }_{2}$ DCFDA in the presence of glucose treatment. Media $\mathrm{pH}$ was maintained at uniform levels throughout the experiment. At the end of the period measured, cultures were fixed with $4 \%$ paraformaldehyde and prepared as described (2). Using a Noran confocal microscope fitted with $\mathrm{Oz}$ with Intervision (Noran Instruments, Madison, WI), a z-series was examined and neuron DCF pixel intensity was measured using a specific image analysis macro written for NIH Image. To measure peak DCF levels, scanned images of each neuron were obtained and average pixel intensity for the neuron and peak intensity in
Mt in the scanned image were measured. As DCF changes were maximal in Mt and showed a stronger association with changes in ROS generation, Mt pixel intensity was used for further analysis. The Mt pixel intensity was averaged and the background fluorescence subtracted. Results were obtained from at least 50 neurons/condition in each of six experiments.

To confirm that glucose generates ROS, dihydroethidium (DHE) conversion to ethidium by oxidation was measured. The ratio of DHE:ethidium was determined using a fluorimeter (Fluoroskan Ascent II, Helsinki, Finland) and standardized against DRG protein. DHE was measured using $355 \mathrm{~nm}$ excitation and $430 \mathrm{~nm}$ emission, and ethidium was measured using $518 \mathrm{~nm}$ excitation and $605 \mathrm{~nm}$ emission. Results were obtained from 200,000 neurons/well using 12-well plates in four separate experiments.

\section{Measurement of Mt size}

DRG neurons were loaded with CMTMRos using confocal microscopy with $540 \mathrm{~nm}$ excitation. Each Mt is a complex fractal-like network, making accurate analysis of volume difficult. Therefore, the maximum cross-sectional area was determined from identification of each individual Mt throughout the z-series. All distinguishable Mt at each step level in the z-series were measured at the largest cross-sectional area of the Mt. The size of the Mt was determined using the perimeter of visible CMTMRos and reflects the Mt area at the measured level. Identical methods of identification and measurement were applied to each Mt in each cell and in each condition, thus reducing the effect of technical artifact. The Mt areas were then averaged for each neuron and, where necessary, results were first converted to a log normal distribution before obtaining descriptive statistics or performing tests of inference about the data set. Results were obtained for $>100 \mathrm{Mt}$ in at least 10 randomly measured neurons per condition. Experiments were repeated at least in triplicate.

\section{Measurement of changes in the $\Delta \Psi_{M}$}

Three different dyes were used for the measurement of $\Delta \Psi_{\mathrm{M}}$ : TMRM ester, CMTMRos, and JC-1. Each dye was applied for $30 \mathrm{~min}$ at $37^{\circ} \mathrm{C}$ prior to analysis and $\mathrm{pH}$ was stable throughout experiments. Uptake of TMRM ester $(50 \mathrm{nM})$ was measured in live DRG using fluorimetry (Fluoroskan Ascent II, Helsinki, Finland) with $485 \mathrm{~nm}$ excitation and $590 \mathrm{~nm}$ emission. Twelve-well plates with 200,000 neurons per well were used. Results were corrected for the DRG protein concentration. Uptake of CMTMRos (50 nM) was determined after washing the cells with fresh media and fixation in $4 \%$ paraformaldehyde. Using the Noran confocal microscope, the mean pixel intensity was obtained for each Mt and background intensity values were subtracted ( $>100 \mathrm{Mt} /$ neuron and 10 neurons/ condition). JC1 (2.5 $\mu \mathrm{g} / \mathrm{mL})$ was measured using confocal microscopy and fluorimetry. Depending on the state of polarization, this cell-permeant dye exists as a red fluorescent aggregate (absorption: $488 \mathrm{~nm}$; emission: $590 \mathrm{~nm}$ ) at negative membrane potentials $(<-100 \mathrm{mV})$ and as a green fluorescent monomer (absorption: $488 \mathrm{~nm}$ emission: $530 \mathrm{~nm}$ ) with more positive membrane potentials $(>-100 \mathrm{mV})$. The 590/ $530 \mathrm{~nm}$ ratios were obtained to characterize the $\Delta \Psi_{\mathrm{M}}$. To establish the validity of TMRM, JC1, and CMTMRos in accurately measuring changes in the $\Delta \Psi_{\mathrm{M}}$ in DRG, neurons were assessed as follows: with $10 \mu \mathrm{M}$ valinomycin, almost complete depolarization was observed; with $10 \mu \mathrm{M}$ oligomycin, there was hyperpolarization in control DRG but rapid depolarization in high glucose-treated DRG with compro- 
mised ATP regulation; the uncoupling agent FCCP $(2 \mu \mathrm{M})$ induced partial depolarization.

\section{Measurement of ADP:ATP ratios}

ATP and ADP levels were measured using a luciferase kit with a luminescence plate reader (Fluroskan Ascent II, Labsystems, Helsinki). Equal numbers of DRG neurons (200,000/well) were cultured in 12-well plates. After exposure to treatment media for a specified time, cells were lysed in the kit's nucleotide-releasing reagent and transferred to 96-well white-walled plates according to instructions. ATP was measured in the sample, then ADP was converted to ATP and the measurement was repeated to permit the calculation of the ratio of ADP:ATP. Results were then corrected for DRG protein concentrations.

Immunohistochemistry for cytochrome $c$ release, caspase-3, caspase-9, and TUNEL staining

To measure cytochrome $c$ translocation, DRG were first incubated in CMTMRos as indicated above to localize the Mt. DRG were then fixed with $4 \%$ paraformaldehyde, permeabilized with Triton X-100, incubated in 1:500 of $0.1 \mathrm{mg} / \mathrm{mL}$ cytochrome $c$ MAb (clone 6H2.B4) for $2 \mathrm{~h}$ at $37^{\circ} \mathrm{C}$, followed by $10 \mu \mathrm{g} / \mathrm{mL}$ FITC labeled secondary anti-mouse at room temperature for $1 \mathrm{~h}$. A Noran confocal microscope was used to view cytochrome $c$ translocation from the Mt.

Fixed DRG neurons were treated with rabbit anti-CM1 antibody (1:5000 of a $0.410 \mathrm{mg} / \mathrm{mL}$ working stock) overnight at room temperature, then with $7.5 \mu \mathrm{g} / \mathrm{mL}$ rhodamineconjugated goat anti-rabbit antibody for $1 \mathrm{~h}$ as described (2). The nuclear chromatin was counterstained with $1 \mu \mathrm{g} / \mathrm{mL}$ bisbenzamide in PBS. The number of apoptotic neurons was determined using a random grid counting system (2) on a Zeiss axiophot 2 microscope (Carl Zeiss, Thornwood, NY) with a $60 \times$ oil immersion objective. Caspase- 9 was detected with the carboxyfluorescein derivative of benzyloxycarbonyl leucylglutamylhistidylaspartic acid fluoromethyl ketone FAMLEHD-FMK (CaspaTag). FAM-LEHD-FMK enters the cell and irreversibly binds to caspase-9, and to a lesser extent to caspase $-4,-5$, and -6 . The percentage of cells with positive specific fluorescence was measured as described above and DRG TUNEL staining was performed (2).

\section{Statistical analysis}

Assumptions about the Gaussian distribution of data and rules for transformation of nonnormative data were made as described (2). Comparison of dependent variables was performed using factorial analysis of variance. For graphic and descriptive purposes, controls are expressed as 100\%, and experimental conditions are referenced as control $\pm \mathrm{SE}$.

\section{RESULTS}

\section{PCD in vitro is dependent on the concentration of glucose}

To determine the optimal culture concentration of glucose, DRG neurons were exposed to increasing concentrations of glucose ranging from $10 \mathrm{mM}$ total glucose to $45 \mathrm{mM}$ and cleavage of caspase-3 was measured (Fig. 1). The lowest level of CM1-positive neurons was observed with 25-30 $\mathrm{mM}$ glucose (Fig. 1). In

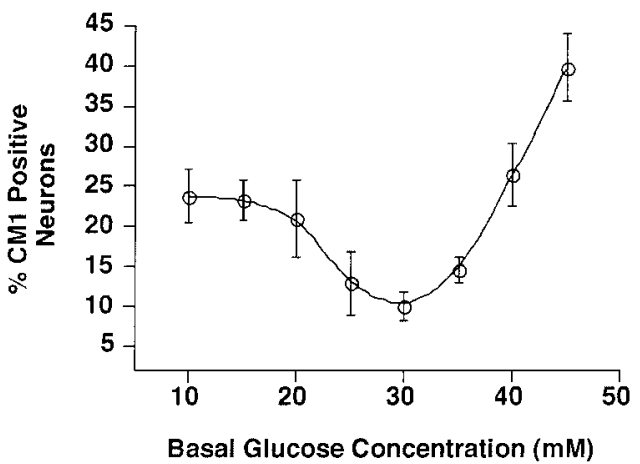

Figure 1. Glucose induces a dose-dependent increase in caspase-3 cleavage. DRG and increasing concentrations of added glucose were cultured for $6 \mathrm{~h}$, then stained for activated caspase-3. Basal glucose indicates the total concentration of glucose in the medium. Optimal neuronal survival is observed with $25-30 \mathrm{mM}$ basal glucose. With glucose concentrations $<25 \mathrm{mM}$ or $>35 \mathrm{mM}$ there is a progressive increase in PCD.

contrast, even small increases in glucose of $10 \mathrm{mM}$ higher $(40 \mathrm{mM})$ resulted in a $16 \%$ increase in the number of CM1-positive neurons $(P<0.001)$. Similarly, concentrations of glucose $<25 \mathrm{mM}$ resulted in increased cleavage of caspase-3.

\section{High glucose induces formation of ROS in DRG neurons}

DCF levels were measured in control neurons and after exposure to $45 \mathrm{mM}$ glucose. With high glucose there was a $75 \%$ increase in the mean level of DCF compared with the corresponding control value (Fig. 2). DCF levels peaked at $3-4 \mathrm{~h}(P<0.001)$. Beyond $5 \mathrm{~h}$, levels of DCF decreased and by $48 \mathrm{~h}$ were slightly below control levels (not statistically significant). With increased concentrations of added glucose of up to $150 \mathrm{mM}$, ROS production increased. An additional $8 \%$ increase in DCF levels with $150 \mathrm{mM}$ glucose vs. $45 \mathrm{mM}$ glucose was observed (NS). With higher concentrations of glucose $(>150 \mathrm{mM})$, DCF levels peaked earlier $(<3 \mathrm{~h})$ and declined by $6 \mathrm{~h}$. An increase in generation of ROS in the cell culture model was confirmed with DHE fluorescence measurements using a fluorimeter. DHE is primarily a measure of superoxide generation. Using measurement of DHE oxidation (ethidium:DHE) ratio, the highest ratio was obtained at $3-4 \mathrm{~h}$, with levels twofold higher than control (Fig. 2B, $P<0.001$ ), followed by a decline in levels by $5-6 \mathrm{~h}$.

\section{Increased glucose induces Mt enlargement}

We have reported that DRG from hyperglycemic rats have an increased number of swollen disrupted Mt (2). The current study examined the time course and extent of glucose-induced neuronal Mt swelling and depolarization in cultured DRG. Mt size was measured using confocal microscopic images of CMTMRostreated DRG neurons. CMTMRos is an aldehyde-fixable 


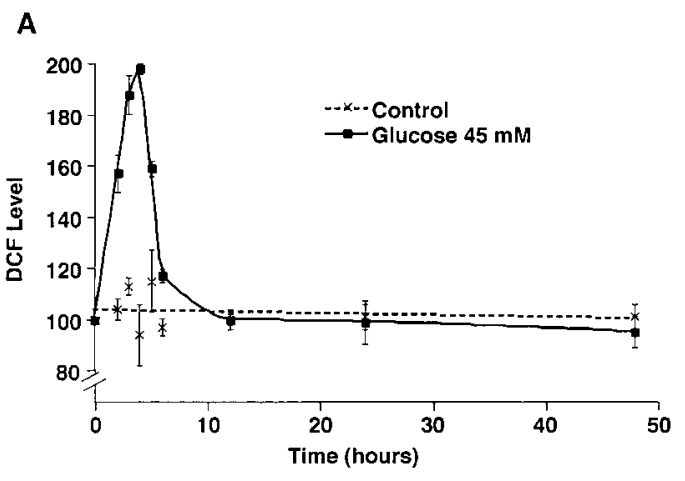

B

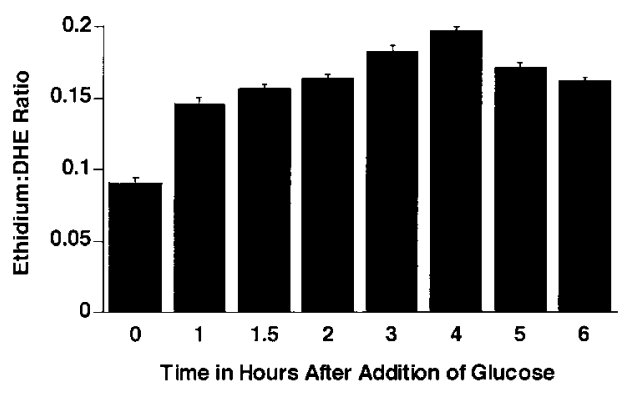

Figure 2. Glucose induces production of ROS. A) DRG \pm 45 $\mathrm{mM}$ glucose were cultured for up to $48 \mathrm{~h}$ in $2 \mu \mathrm{M} \mathrm{CM}$ $\mathrm{H}_{2}$ DCFDA. Fluorescent DCF was measured using confocal microscopy. Results were obtained from 5 experiments standardized against control values at $0 \mathrm{~h}$. With added glucose, results are expressed as \% control at each time point (1-48 h). In control media (containing $25 \mathrm{mM}$ glucose), results at $1-48 \mathrm{~h}$ are expressed as $\%$ control $0 \mathrm{~h}$. There was no statistical change in control values after $48 \mathrm{~h}$. In $45 \mathrm{mM}$ glucose there was an initial increase in the production of ROS (mean DCF levels) that peaked at 3-4 h, then declined, corresponding to increased neuronal death. B) DRG neurons in culture were exposed to $45 \mathrm{mM}$ glucose for $0-6 \mathrm{~h}$. DHE $(3 \mu \mathrm{M})$ oxidation to ethidium was measured using fluorimetry to determine superoxide production during peak glucose-induced ROS generation. Results are expressed as the ethidium:DHE ratio. The ratio in controls remained at the $0 \mathrm{~h}$ value for the next $6 \mathrm{~h}$.

dye that allows measurement of Mt size and accurately reflects the $\Delta \Psi_{\mathrm{M}}$ in $\mathrm{Mt}(11)$. In the presence of $45 \mathrm{mM}$ glucose, the Mt were significantly enlarged compared with control neurons. At $6 \mathrm{~h}$ the mean $\mathrm{Mt}$ area was $0.43 \pm 0.03 \mu \mathrm{m}^{2}$ for control DRG and $0.66 \pm 0.08 \mu \mathrm{m}^{2}$ for Mt from DRG treated with $45 \mathrm{mM}$ glucose. In the presence of this high glucose, mean Mt size (measured Mt area) increased by $50 \%$ at $6 \mathrm{~h}$ (Fig. 3) and by $>100 \%$ at $24 \mathrm{~h}(P<0.001)$. At $6 \mathrm{~h}$ vs. $48 \mathrm{~h}$, there is greater variability in Mt size with high glucose. This is due to differences in degree of swelling of individual $\mathrm{Mt}$ as indicated by the larger SE at $6 \mathrm{~h}$ vs. $48 \mathrm{~h}$ (Fig. 3). By $48 \mathrm{~h}$, all the Mt are enlarged; there is less variability and thus a progressive increase in mean Mt size. Increasing concentrations of added glucose (45-300 mM) induce a dose-dependent enlargement of Mt in DRG neurons. With $150 \mathrm{mM}$ glucose there is a $130 \%$ increase in mean Mt size; with $300 \mathrm{mM}$ glucose, mean Mt size more than doubles (166\% increase) at $6 \mathrm{~h}$ after exposure to high glucose $(P<0.0001)$.

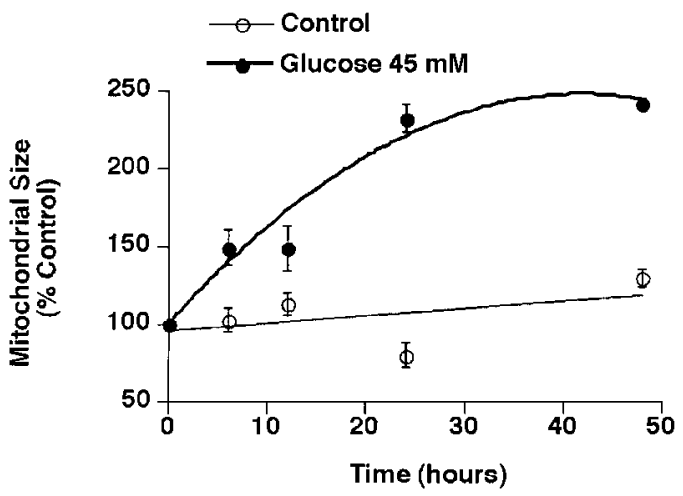

Figure 3. High Glucose induces a dose-dependent enlargement of DRG Mt and Mt membrane depolarization. In $45 \mathrm{mM}$ glucose there is a progressive increase in mean Mt size up to $48 \mathrm{~h}$. DRG neurons were treated with $5 \mu \mathrm{M}$ mitotracker orange, fixed, and quantified by confocal microscopy. Values for control neurons are plotted as $\%$ control at time $0 \mathrm{~h}$. Values for DRG treated with added glucose are plotted as $\%$ of corresponding control at time points $0-48 \mathrm{~h}$. Plotted values represent mean $\pm \mathrm{SE}$.

\section{Glucose-induced changes in the $\Delta \Psi_{M}$}

The change in $\Delta \Psi_{\mathrm{M}}$ was assessed in DRG neurons treated with high glucose using TMRM and fluorimetry (Fig. 4). There was an initial twofold increase in the $\Delta \Psi_{\mathrm{M}}$ at $3 \mathrm{~h}$, followed by a threefold decrease in the $\Delta \Psi_{\mathrm{M}}$ at $6 \mathrm{~h}(P<0.001)$. Depolarization continued for up to 24 h. In Fig. 4, the small SE values reflect the large number of samples used in the analysis. Using confocal measurement of CMTMRos, there was further evidence of MMD at $6 \mathrm{~h}$, with a decrease in CMTMRos levels to $66.4 \pm 7.7 \%$ of control $(P<0.001)$. These results were further confirmed with JC1 fluorimetry. The 590/530 ratio was 5.6 in control DRG neurons and 3.92 (70\% of control) in $45 \mathrm{mM}$ glucose at $6 \mathrm{~h}$. Using confocal microscopy of JC1, the $590 / 530$ ratio was $46 \pm 9 \%$ of control at $6 \mathrm{~h}(P<0.001)$.

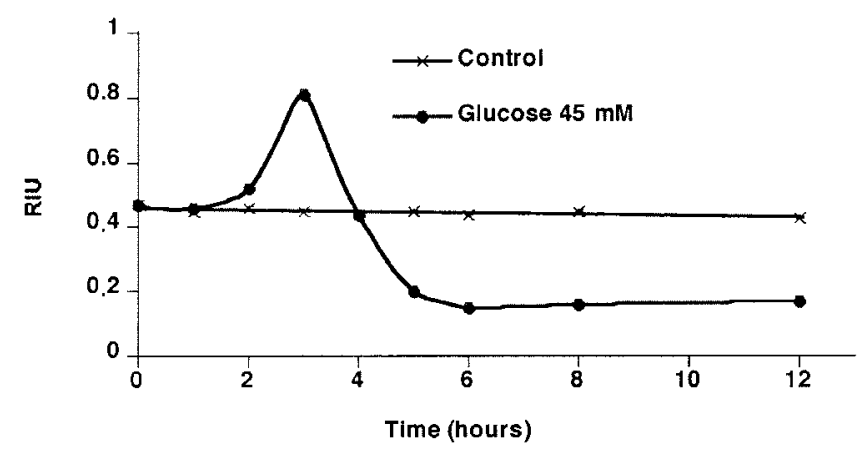

Figure 4. High glucose induces initial hyperpolarization, followed by depolarization of the $\Delta \Psi_{\mathrm{M}}$. The graph indicates the change in TMRM fluorescence (relative intensity units, RIU) in DRG neurons in $45 \mathrm{mM}$ or control (25 mM) glucose as a $\%$ of control. Fluorescence was measured using fluorimetry and represents data from 4 experiments. Peak hyperpolarization is seen at $3 \mathrm{~h}$, followed by depolarization $5 \mathrm{~h}$ after exposure to high glucose. There was no significant change in TMRM fluorescence in control neurons during this period. 


\section{Glucose increases the ADP:ATP ratio}

When DRG were exposed to an increased glucose load there was a rapid increase in the ADP:ATP ratio at $0.5 \mathrm{~h}$ to $2.74 \pm 0.23$, with a decrease in absolute levels of ATP (Fig. 5). By $1 \mathrm{~h}$ the ratio had started to fall and was accounted for mainly by a fall in absolute ATP levels, to 0.13 chemiluminescent units vs. 0.32 units in the control DRG $(P<0.0001)$. The decrease in the ADP:ATP ratio corresponded to hyperpolarization of the $\Delta \Psi_{\mathrm{M}}$. The decrease in the ADP:ATP ratio continued until $5 \mathrm{~h}$, followed by an increase in the ratio corresponding to depolarization on the $\Delta \Psi_{\mathrm{M}}$. In control neurons, the ADP:ATP ratio remained stable at the $0 \mathrm{~h}$ level throughout the experiment.

In DRG neurons exposed to glucose concentrations of $<25 \mathrm{mM}$ such as relative "hypoglycemia," there was a decrease in absolute ATP levels. DRG neurons were cultured in $5 \mathrm{mM}$ glucose or $25 \mathrm{mM}$ (control) conditions over $6 \mathrm{~h}$. By $3 \mathrm{~h}$ there was a $60 \%$ decrease in total ATP levels compared with control neurons (mean of 3 separate experiments).

High glucose induces cytochrome $c$ translocation from the Mt to the cytosol

The next series of experiments addressed the question of whether high glucose induces translocation of cytochrome $c$ from the Mt to the cytosol, an initiator event in PCD (1). Colocalization of CMTMRos and cytochrome $c$ was used to visualize Mt containing cytochrome $c$ (Fig. 6). In control neurons, cytochrome $c$ colocalizes with the Mt (Fig. $6 a$ ). In the presence of 45 $\mathrm{mM}$ glucose, the Mt are enlarged and there is translocation of cytochrome $c$ to the cytosol (Fig. 6b), consistent with initiation of PCD. As opposed to epifluorescent microscopy, with high magnification confocal microscopy released cytochrome $c$ is visualized as dis-



Figure 5. Effect of high glucose on the ADP:ATP ratio. The graph indicates time-related changes in the ADP:ATP ratio after exposure to $45 \mathrm{mM}$ glucose for $0-12 \mathrm{~h}$. The $0 \mathrm{~h}$ time point is representative of the control ADP:ATP ratio, which remained stable over $12 \mathrm{~h}$. Results are representative of 4 separate experiments.
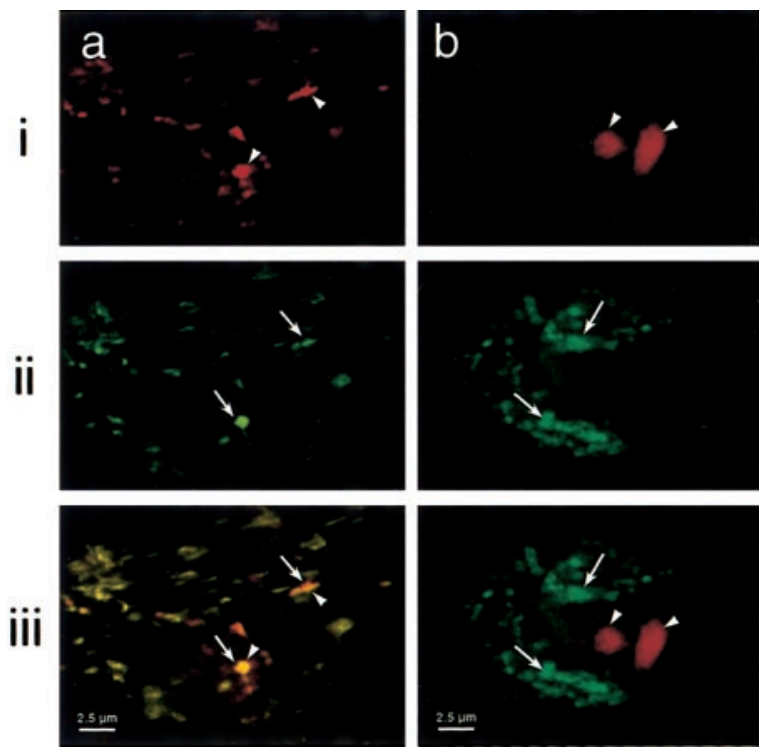

Figure 6. High glucose induces cytochrome $c$ translocation from the Mt to the cytosol. DRG cultures were treated for $6 \mathrm{~h}$ with a) control media (25 mM glucose) or b) $45 \mathrm{mM}$ glucose. The top row $(a \mathrm{i}-b \mathrm{i})$ indicates Mt staining (red with white arrowheads) with CMTMRos in single DRG neurons, the second row (aii-bii) indicates cytochrome $c$ staining (green with white arrows), and the third row (aiii-biii) overlay of cytochrome $c$ and Mt. Mt (white arrowheads) and cytochrome $c$ staining (white arrows) are superimposed (yellow), indicating that cytochrome $c$ is maintained in the Mt (normal state) (aiii). Functional Mt are more numerous and smaller in the control neuron. biii) Mt exposed to high glucose for $6 \mathrm{~h}$ (white arrowheads) are enlarged and fewer compared with control. Cytochrome $c$ (white arrows) has been released from the Mt into the cytosol, consistent with initiation of PCD.

crete staining, possibly due to colocalization with the apoptosome.

\section{Glucose induces caspase-3 cleavage and apoptosis}

Having established that glucose mediates ROS accumulation and Mt swelling in DRG, we next asked whether glucose-induced caspase- 3 cleavage and apoptosis in DRG neurons. In the presence of $45 \mathrm{mM}$ glucose, punctate positive CM-1 antibody staining was observed in the cytosol of DRG neurons exposed to excess glucose, consistent with the presence of caspase-3 cleavage (Fig. 7A). This was coupled with evidence of chromatin condensation in DRG nuclei, consistent with apoptosis (Fig. 7A). There was a progressive increase in caspase-3 cleavage after exposure to $45 \mathrm{mM}$ glucose. By $6 \mathrm{~h}$ the cleaved caspase-3 level had increased by $40 \%$ (Fig. $7 B$ ) compared with timed controls $(P<0.0001)$, corresponding to the time point of maximal MMD. In comparison, there was no statistical difference between control caspase-3 levels at 0-24 h. The pan-caspase inhibitor BAF inhibited neuronal cell death in the presence of high glucose (Fig. 7C). With $45 \mathrm{mM}$ glucose there was a $36 \%$ increase in the number of apoptotic neurons above control $(P<0.01)$ and a $66 \%$ increase with $150 \mathrm{mM}$ added glucose $(P<0.001$, data 
A.

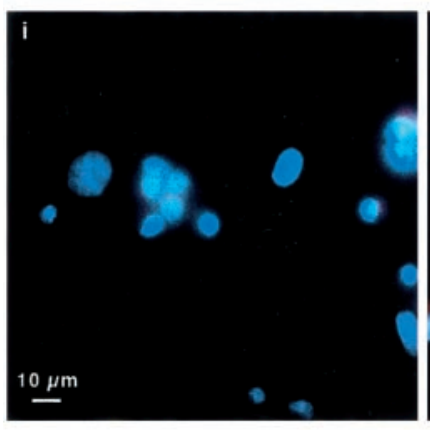

B.

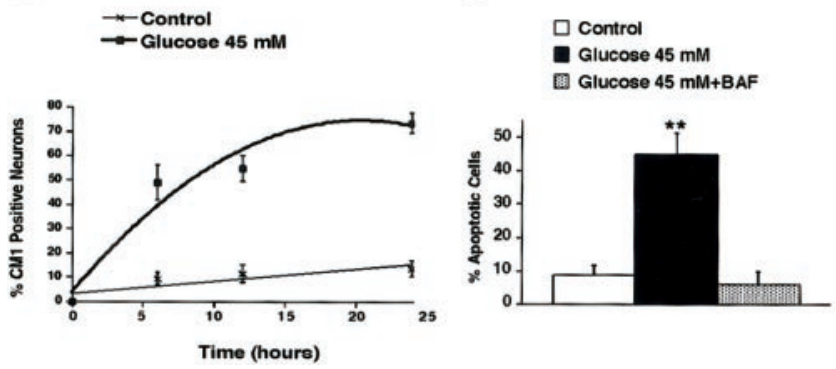

Figure 7. Increasing concentrations of glucose induce cleavage of caspase-3 and apoptosis that is blocked by pancaspase inhibitors. A) Increased glucose induces cleavage of caspase-3 in cultured DRG neurons. DRG neurons were cultured in i) control media or ii) media containing $45 \mathrm{mM}$ glucose. After $6 \mathrm{~h}$, the cultures were fixed and stained with $5 \mu \mathrm{g} / \mathrm{mL} \mathrm{CM} 1$ to indicate cleaved (active) caspase-3 (orange-red), and 1 $\mu \mathrm{g} / \mathrm{mL}$ bisbenzamide to indicate nuclear chromatin (blue). i) Immunofluorescent image showing normal diffuse nuclear staining in the DRG neuron (blue). ii) Immunofluorescent image showing cleaved caspase-3 in the cytoplasm (white arrows) with some of the nuclei showing aggregation of chromatin consistent with apoptosis (white arrowheads). Panel ii shows high magnification of a single DRG neuron with punctate diffuse CM1 staining (cleaved caspase-3) in the cytoplasm, with chromatin clumping consistent with dissolution of the nucleus (white arrowhead). B) High glucose induces activation of caspase-3 in neurons. Cultured DRG neurons were exposed to $45 \mathrm{mM}$ added glucose for $0-24 \mathrm{~h}$. Neurons were stained with the CM1 antibody, which measures cleaved caspase- 3 in the neuron. The \% of CM1-positive neurons increases with time up to $24 \mathrm{~h}$. In comparison there was no significant change in controls from 0 to $24 \mathrm{~h} . C$ ) Inhibition of the cysteine protease $\mathrm{PCD}$ cascade by BAF prevents high glucose-induced apoptosis in rat DRG neurons. Dissociated DRG neurons cultured in defined serum-free media were exposed for $24 \mathrm{~h}$ to high glucose $\pm 100 \mu \mathrm{M}$ BAF. The $\%$ apoptotic neurons increased to $45 \%$ with $45 \mathrm{mM}$ glucose. With $100 \mu \mathrm{M}$ BAF there was complete inhibition of neuronal cell death. ${ }^{*} P<0.01$.

not shown). In contrast, $100 \mu \mathrm{M}$ BAF maintained the percent apoptotic neurons at control levels even in the presence of high glucose.

\section{BKA inhibits Mt membrane depolarization} and caspase-3 cleavage

The next series of experiments determined whether inhibition of the adenine nucleotide translocase/volt- age-dependent anion channel (ANT/VDAC) complex on the Mt membrane prevented Mt swelling, caspase cleavage, and cell death. BKA is an inhibitory ligand for the Mt ANT (12) and causes prolonged inhibition of MMD. To determine whether stabilization of the $\Delta \Psi_{\mathrm{M}}$ in the presence of high glucose prevented cleavage of caspase-3 and PCD, DRG neurons were treated with 100 $\mu \mathrm{M}$ BKA. Data were standardized against control samples that represent $100 \%$ (Fig. 8A). There was a $54 \%$ increase in the mean Mt size at $6 \mathrm{~h}$ with $45 \mathrm{mM}$ glucose compared with control $(P<0.001)$. In the presence of $45 \mathrm{mM}$ glucose, $100 \mu \mathrm{M}$ BKA inhibited Mt swelling, maintaining mean Mt size at control levels. After $24 \mathrm{~h}$, BKA still inhibited Mt swelling induced by $45 \mathrm{mM}$ added glucose (Fig. 8A), but less effectively than at $6 \mathrm{~h}$. There was no significant difference in Mt size in control DRG neurons $\pm 100 \mu \mathrm{M}$ BKA.

In contrast to Mt size, measurements of Mt pixel intensity revealed a reduction in Mt CMTMRos intensity reflecting loss of the $\Delta \Psi_{\mathrm{M}}$ with high glucose (11). There was a $15 \%$ reduction in the Mt intensity (Fig. $8 B$ ) compared with control Mt intensity at $6 \mathrm{~h}(P<0.05)$ and a $32 \%$ reduction at $24 \mathrm{~h}(P<0.001)$. Thus, $45 \mathrm{mM}$ glucose-induced progressive MMD. In contrast, addition of $100 \mu \mathrm{M}$ BKA completely inhibited MMD induced by $45 \mathrm{mM}$ glucose at $6 \mathrm{~h}$. At $24 \mathrm{~h}$, BKA partially inhibited MMD, but was not statistically different from control values. As expected from inhibition of the ANT, BKA induces mild hyperpolarization of the Mt membrane in control neurons, but stabilization of the $\Delta \Psi_{\mathrm{M}}$ prevents subsequent depolarization observed with high glucose alone (Fig. $8 B$ ). Even with redosing at $12 \mathrm{~h}$, BKA less effectively inhibited Mt swelling and MMD at $24 \mathrm{~h}$ vs. $6 \mathrm{~h}$. The initial rise in caspase-3 cleavage with added glucose was blocked by BKA at $6 \mathrm{~h}$. With $45 \mathrm{mM}$ added glucose and BKA, there were only $6 \%$ apoptotic neurons vs. $3 \%$ with $45 \mathrm{mM}$ added glucose alone $(P<0.001)$ and $7 \%$ in control (Fig. $8 C)$.

To determine whether BKA inhibition of PCD was independent of ROS generation, DCF levels were measured using confocal microscopy. BKA increased generation of ROS. Mean DCF levels were 26\% higher with high glucose and BKA than with high glucose alone. Under control conditions, DCF levels were $31 \%$ higher in the presence of BKA (mean of 3 separate experiments).

\section{Myxothiazole inhibits glucose-induced oxidative stress and Mt dysfunction}

To determine whether inhibition of ROS generation prevents Mt dysfunction and initiation of PCD in the presence of high glucose, the generation of Mt oxidative species was blocked using myxothiazole. Myxothiazole inhibits the Mt respiratory chain at cytochrome $b-c_{1}$ (13). In the presence of $45 \mathrm{mM}$ added glucose, there was a $20 \%$ increase in the mean DCF level in DRG neurons at $6 \mathrm{~h}(P<0.001)$, indicating increased production of ROS (Fig. 9A). At $12 \mathrm{~h}$, DCF had returned to control levels as indicated in Fig. 2. At $6 \mathrm{~h}$ in the 
A
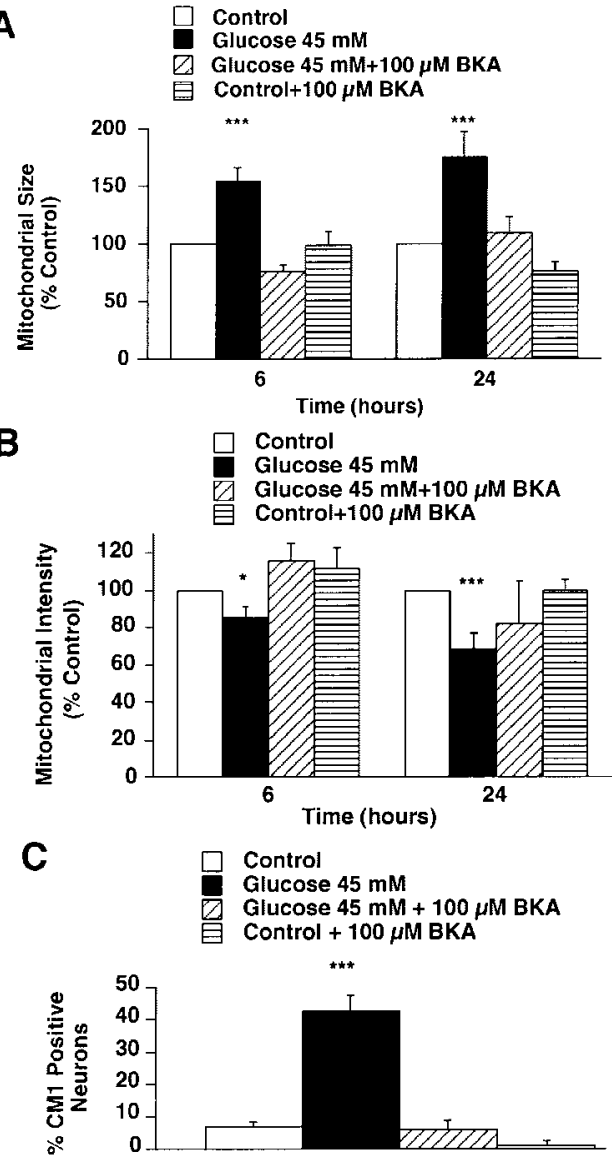

Figure 8. Inhibition of Mt membrane depolarization by BKA inhibits Mt enlargement and caspase cleavage. A) BKA inhibits Mt swelling in DRG neurons induced by $45 \mathrm{mM}$ added glucose. DRG neurons were treated with $45 \mathrm{mM}$ glucose \pm $100 \mu \mathrm{M}$ BKA for $6-24 \mathrm{~h}, 5 \mu \mathrm{M}$ CMTMRos for $30 \mathrm{~min}$, then Mt size was measured. With $45 \mathrm{mM}$ glucose at $6 \mathrm{~h}$ there was a $54 \%$ increase in mean Mt size and a $75 \%$ increase at $24 \mathrm{~h}$ compared with controls $(P<0.001)$. Glucose-induced enlargement of Mt was blocked by BKA. There was no statistically significant differences between control, control + BKA, and glucose + BKA at $24 \mathrm{~h}$. B) BKA inhibits loss of Mt intensity dependent on the $\Delta \Psi_{\mathrm{M}}$. With $45 \mathrm{mM}$ glucose, there was a $15-32 \%$ decrease in mean Mt intensity compared with control between 6 and $24 \mathrm{~h}$. BKA maintained Mt intensity at control levels at $6 \mathrm{~h}$ consistent with inhibition of the ANT/VDAC complex and maintenance of the $\Delta \Psi_{\mathrm{M}}$. By $24 \mathrm{~h}$ BKA less effectively inhibited glucose-induced MMD and mean Mt intensity was reduced but this was not statistically significantly different from control levels. $C$ ) BKA blocks glucose-induced cleavage of caspase-3 in DRG neurons measured by CM1 antibody staining. DRG neurons were treated with $45 \mathrm{mM}$ extra glucose $\pm 100 \mu \mathrm{M}$ BKA for $6 \mathrm{~h}$. BKA acts by blocking the ANT/VDAC complex of the Mt and prevents loss of the $\Delta \Psi_{\mathrm{M}}$. In the presence of BKA there is reduced CM-1 staining even in the presence of $45 \mathrm{mM}$ glucose, indicating that inhibition of Mt depolarization is critical in preventing PCD. For all graphs, $* P<0.05, * * * P<0.001$.

presence of $45 \mathrm{mM}$ glucose, $3 \mu \mathrm{M}$ myxothiazole maintained the mean DCF at $98.2 \pm 3.3 \%$ of control (Fig. $9 A)$. The DCF level with high glucose and myxothiazole was significantly below DCF levels with $45 \mathrm{mM}$ glucose alone $(P<0.001)$.

As previously observed, $45 \mathrm{mM}$ glucose increased mean Mt size progressively over $24 \mathrm{~h}$. In contrast, 3 $\mu \mathrm{M}$ myxothiazole inhibited Mt swelling; even at $24 \mathrm{~h}$, compared with controls, the mean Mt size was not statistically different (Fig. 9B). Myxothiazole also stabilized the $\Delta \Psi_{\mathrm{M}}$. Using $45 \mathrm{mM}$ glucose and CMTMRos, mean Mt intensity decreased by $34 \%$ at $6 \mathrm{~h}$ $(P<0.001)$ and $39 \%$ at $24 \mathrm{~h}(P<0.001)$, consistent with MMD. In contrast, when myxothiazole was added to high glucose, Mt intensity remained at $102 \%$ of control at $6 \mathrm{~h}$ and $104 \%$ at $24 \mathrm{~h}$. Using TMRM and $45 \mathrm{mM}$ glucose, myxothiazole blocked initial glucose-induced Mt membrane hyperpolarization: TMRM levels were $3 \%$ below control values at the peak of glucose-induced hyperpolarization $(3 \mathrm{~h})$. Subsequently, myxothiazole prevented MMD observed with high glucose at $6 \mathrm{~h}$, probably due to initial ATP synthase reversal. In both control and high glucose-treated cultures, addition of myxothiazole eventually led to depolarization of the $\Delta \Psi_{\mathrm{M}}$ at $24 \mathrm{~h}$.
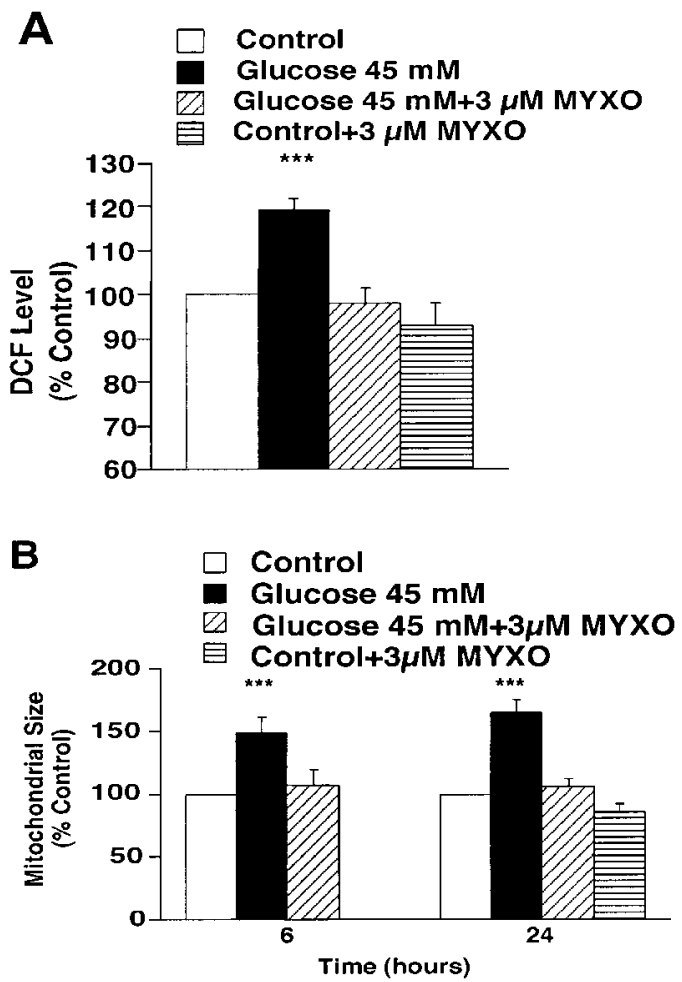

Figure 9. Inhibition of Mt ROS generation by myxothiazole blocks Mt enlargement and depolarization. A) Myxothiazole inhibits the production of Mt ROS. DCF levels are expressed as $\%$ of the corresponding control value. In the presence of $45 \mathrm{mM}$ glucose, there was a $20 \%$ increase in the mean DCF level in DRG neurons at $6 \mathrm{~h}(P<0.001)$, indicating increased production of ROS. At $6 \mathrm{~h}, 3 \mu \mathrm{M}$ myxothiazole in the presence of $45 \mathrm{mM}$ glucose maintained the mean DCF at $98.2 \pm 3.3 \%$ of control $(P<0.001)$. There was no difference in DCF levels between control and control \pm myxothiazole. $B$ ) Myxothiazole inhibits Mt enlargement at 6 and $24 \mathrm{~h}$ in DRG neurons with high glucose. In the presence of $45 \mathrm{mM}$ glucose, $3 \mu \mathrm{M}$ myxothiazole maintains Mt size at $107 \%$ of control values at $6 \mathrm{~h}$ and $106 \%$ of control values at $24 \mathrm{~h}$ (not significantly different from control). 
Myxothiazole inhibits glucose-induced caspase-3 and -9 cleavage

Myxothiazole also inhibited cleavage of upstream initiator caspase-9 (Fig. 10A), which is released early in initiation of PCD $(1,9)$. With $45 \mathrm{mM}$ glucose, there was increased binding of the carboxyfluorescein substrate FAM-LEHD-FMK, which preferentially binds to active caspase- 9 and not caspase-3. Cleavage of caspase-9 was blocked by $3 \mu \mathrm{M}$ myxothiazole compared with control $(P<0.001)$ (Fig. 10B). At both 6 and $24 \mathrm{~h}, 3 \mu \mathrm{M}$ myxothiazole inhibited cleavage of caspase- 3 induced by $45 \mathrm{mM}$ glucose (Fig. 10C). At $6 \mathrm{~h}$, caspase- 3 cleavage in glucose + myxothiazole conditions was not statistically different from controls. At $24 \mathrm{~h}$, myxothiazole

A.
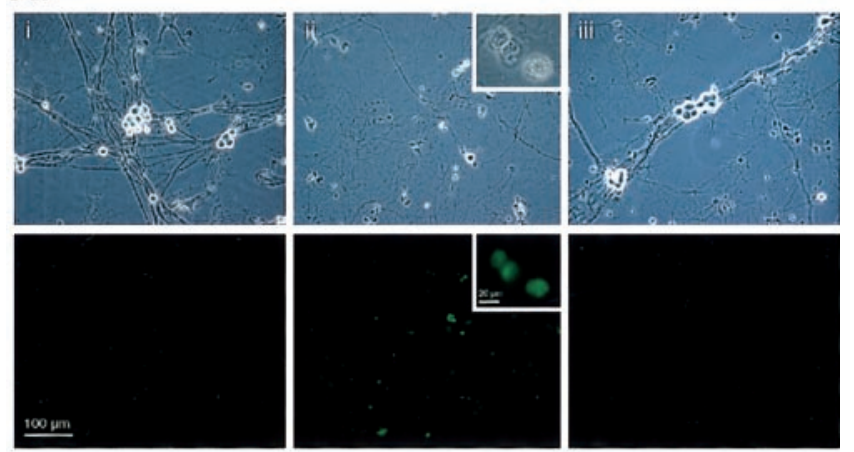

B.

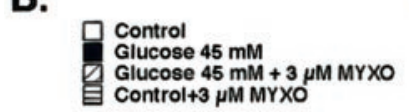

C.

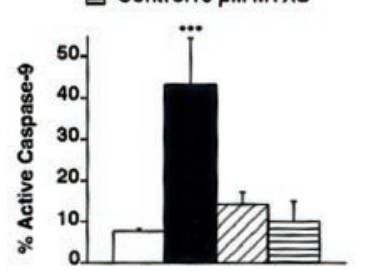



Figure 10. The cytochrome $b-c_{1}$ inhibitor myxothiazole blocks cleavage of caspase-3 and -9. A) DRG neurons were cultured in i) control media (25 mM glucose), ii) $45 \mathrm{mM}$ glucose, or iii) $45 \mathrm{mM}$ glucose and $3 \mu \mathrm{M}$ myxothiazole for $6 \mathrm{~h}$. The image shows phase-contrast images (top row) and matched epifluorescent images (bottom row). With added glucose there was an increase in measured cleaved caspase-9 (ii) compared with control DRG neurons treated with myxothiazole. B) With 45 $\mathrm{mM}$ added glucose at $6 \mathrm{~h}, \%$ caspase-9-positive DRG neurons was increased to $43 \%$ vs. $8 \%$ in control neurons with $25 \mathrm{mM}$ glucose $(P<0.001)$ and $14 \%$ with added myxothiazole. There were no statistical differences between control and myxothiazole-treated groups. C) The \% caspase-3-positive DRG neurons were determined using CM1 antibody at 6-24 h. Compared with control, there is a $41 \%$ increase in caspase-3positive neurons with $45 \mathrm{mM}$ glucose at $6 \mathrm{~h}$ and $56 \%$ at $24 \mathrm{~h}$. Caspase- 3 activation with $45 \mathrm{mM}$ added glucose and myxothiazole was marginally above control at $6 \mathrm{~h}$ (not statistically significant). At $24 \mathrm{~h}$ there was only partial inhibition of caspase- 3 cleavage by myxothiazole $(13 \%>$ control, $P<0.05)$. For all graphs, $* P<0.05, * * * P<0.001$. significantly reduced glucose-induced caspase-3 cleavage, but levels were slightly above those seen in the controls $(P<0.05)$.

\section{TTFA inhibits glucose-induced Mt dysfunction and PCD}

To determine whether inhibition of Mt complexes other than complex III blocked Mt enlargement and caspase activation, DRG neurons cultured in $45 \mathrm{mM}$ glucose were treated with the Mt complex II inhibitor TTFA (Succinate-Q Reductase). TTFA blocked MMD more effectively than Mt enlargement. In $45 \mathrm{mM}$ glucose, there was an increase in Mt size to $166 \%$ of control $(P<0.001)$, but $5 \mu \mathrm{M}$ TTFA maintained Mt size at $121 \%$ of control values at $6 \mathrm{~h}(P<0.01$ compared with control). With $45 \mathrm{mM}$ added glucose, there was a $31 \%$ decrease in mean Mt intensity at $6 \mathrm{~h}$ consistent with depolarization $(P<0.001)$. TTFA partially blocked glucose-induced Mt membrane depolarization to $93 \%$ of control at $6 \mathrm{~h}$ (not significantly different from control). TTFA alone did not significantly alter control Mt intensity levels. TTFA blocked caspase- 3 cleavage up to $24 \mathrm{~h}$ : control: $8 \%$; $45 \mathrm{mM}$ glucose: $30 \%$; glucose + TTFA: $10 \%$ (NS compared with control).

\section{DISCUSSION}

In the current study, glucose induces a dose-dependent effect on cleavage of caspases. With a total in vitro concentration of $25-30 \mathrm{mM}$ glucose, there is optimal DRG neuronal survival. This relatively high concentration of basal glucose most likely reflects the increased energy requirements and metabolic rate of DRG compared with nonneuronal cells. Glucose concentrations $<25 \mathrm{mM}$ rapidly induce ATP depletion and an increase in caspase-3 cleavage. Use of $25 \mathrm{mM}$ glucose in vitro is consistent with requirements reported for central and peripheral nervous system neuronal cultures $(2,14,15)$ but higher than in vivo levels, where high metabolic requirements are maintained by continuous perfusion of neurons and large glucose reserves. Similar to results with reduced glucose levels, basal glucose concentrations $>25 \mathrm{mM}$ induce PCD. These studies confirm our previous findings in animal and cell culture models of diabetes using TUNEL staining $(2,3)$. Thus, in vitro as in vivo, variations in basal glucose as small as $10 \mathrm{mM}$ induce neuronal injury; however, unlike in vivo studies, the in vitro system permits detailed mechanistic studies of glucose generated oxidative stress.

As expected from the evidence of PCD in DRG neurons, there is loss of $\sim 14-21 \%$ of total DRG neurons in diabetic rats $(2,7)$, although there is variation between affected animals and the result does not reach statistical significance. However, if only the largest DRG neurons are assessed in chronically diabetic animals, there is loss of $43 \%$ of the largest neurons in diabetic compared with nondiabetic animals (8). This corre- 
sponds to loss of $53 \%$ of the largest myelinated fibers in chronically diabetic animals (7). Our own findings indicate an increase in oxidative injury in DRG neurons in diabetic rats, providing a pervasive argument that increased ROS is associated with neuronal loss and development of sensory neuropathy. In the current study, high glucose generates ROS coupled with hyperpolarization of the $\Delta \Psi_{\mathrm{M}}$, followed by MMD, which is temporally related to an increase in ADP:ATP ratios and an absolute decrease in ATP levels. This in turn is coupled with cytochrome $c$ release from the intermitochondrial membrane space and cleavage of caspase- 3 and -9 , resulting in DRG apoptosis. Histological Mt disruption and induction of PCD by high glucose have been confirmed in vivo in DRG neurons and are not related to hyperosmolarity at the concentration used in this study ( $45 \mathrm{mM})$ (2). Whereas high glucose increases ROS and destabilizes the $\Psi_{\mathrm{M}}$, inhibitors of the Mt electron transfer chain or of proton transfer block oxidative stress, MMD, and PCD induced by high glucose. This concept is supported in neurons by recent findings in bovine aortic endothelial cells (13); increased cellular production of ROS occurs primarily in the Mt through the electron transport chain, and ROS generation is a causal link between high glucose and PCD.

Increased metabolic flux in the Mt due to high glucose, coupled with reduction of NAD to NADH, results in increased formation of ROS such as superoxides, peroxinitrites, and highly reactive hydroxyl radicals (9). These ROS are associated with membrane lipid peroxidation, nitration of proteins, including tyrosine residues, and degradation of DNA, all of which are associated with induction of apoptosis $(1,2)$. Accumulation of NADH coupled with failure of the Mt creatine phosphate pump to regenerate ATP from ADP also results in disruption of the Mt electron transfer chain and depletion of ATP (13) associated with apoptosis. In this study, there was an initial increase in ADP:ATP ratios and a decrease in total ATP $1 \mathrm{~h}$ after exposure to high glucose. The initial ATP decline is followed by an increase in ATP levels along with a decrease in ADP: ATP ratios at $3 \mathrm{~h}$. This increase in ATP levels is coupled with Mt hyperpolarization, presumably due to increased proton pumping across the inner Mt membrane $(13,16,17)$. By $6 \mathrm{~h}$, ATP levels are depleted, similar to changes in ATP observed previously in chronic neuropathy in diabetic animals (18). This increase in the ADP:ATP ratio at $6 \mathrm{~h}$ corresponds to MMD and initiation of PCD. In contrast to these late events, early Mt membrane hyperpolarization corresponds to peak generation of ROS. With an increase in proton gradient, the electrochemical potential will prolong the life of superoxide-generating electron transport intermediates such as ubisemiquinone (13). Thus, hyperpolarization of the inner Mt membrane coupled with impaired creatine phosphate pumping of ADP will disrupt the electron transfer chain and increase ROS generation.

One of the key events coupled with ROS generation is Mt swelling. In this study, with $45 \mathrm{mM}$ glucose, there is a $50 \%$ increase in mean Mt size at $6 \mathrm{~h}$, corresponding to the peak increase in ROS. Confocal microscopy of serial sections of the DRG and previous electron microscopy (2) indicate there is true Mt enlargement and not simply Mt fusion. Furthermore, the size of control and high glucose-treated Mt measured in this study matches the increase in Mt size observed in vivo using transmission electron microscopy $(2,19)$. Mt swelling is associated with a reduction in the mean $\Delta \Psi_{\mathrm{M}}$. Mt permeability transition occurs with opening of the ANT/VDAC channel spanning the inner and outer Mt membranes. This results in swelling, which in turn disrupts the integrity of the outer Mt membrane (20). These events culminate in MMD and release of proapoptotic factors such as cytochrome $c$ into the cytoplasm that activate the caspase cascade (1). Inhibition of the ANT/VDAC channel also inhibits flux of anions across the inner Mt membrane, which initially stabilizes the $\Delta \Psi_{\mathrm{M}}$ but will eventually disrupt chemiosmotic homeostasis in the Mt, and deplete the cell of ATP (12). In this study, Mt swelling and reduction in the $\Delta \Psi_{\mathrm{M}}$ induced by high glucose are both blocked by BKA, which inhibits opening of the ANT and stabilizes the inner Mt membrane. In the presence of high glucose, BKA inhibits downstream cleavage of caspase- 3 , indicating that glucoseinduced MMD and swelling precede cleavage of caspases. Unlike cyclosporin, BKA is a specific ligand for the ANT that inhibits opening of the ANT (21). Even though this induces mild hyperpolarization of the $\Delta \Psi_{\mathrm{M}}$, BKA works primarily by stabilizing the inner Mt membrane (21), thus confirming that MMD is associated with PCD. As expected, the mild hyperpolarization induced by BKA is associated with an increase in ROS generation; thus, BKA inhibition of PCD at $6 \mathrm{~h}$ is independent of its effect on ROS. At $24 \mathrm{~h}$, BKA is less effective in inhibiting PCD, consistent with a proapoptotic induction of ROS and depletion of ATP.

Although stabilization of the $\Delta \Psi_{\mathrm{M}}$ is able to temporarily block PCD, more effective inhibition of $\mathrm{PCD}$ is achieved by regulation of the Mt electron transfer chain. Four enzyme complexes (I-IV) permit transfer of electrons through the transport chain. In the current study, inhibition of Mt complex III at cytochrome $b-c_{1}$ by myxothiazole (13) not only blocked glucose-induced generation of ROS such as peroxinitrites, but also prevented activation of initiator and effector caspases at $6 \mathrm{~h}$. Myxothiazole blocks electron transport by inhibiting electron flow in the $Q$ cycle. Though this will reduce ATP synthesis, neurons have sufficient glycolytic capacity to survive for at least $6 \mathrm{~h}$. More important, myxothiazole is known to block $Q$ cycle/complex IIImediated ROS production when Mt are oxidizing complex II substrates. Prevention of PCD is most likely explained by the ability of myxothiazole to inhibit ROS generation coupled with prevention of initial Mt hyperpolarization, followed by rapid depolarization. Inhibition of MMD by myxothiazole is probably related to reversal of ATP synthase. This is supported by the finding that oligomycin, which inhibits ATP synthase, 
causes MMD after $6 \mathrm{~h}$ when used in the presence of myxothiazole in DRG. At $6 \mathrm{~h}$, myxothiazole blocks glucose-induced cleavage of caspase- 9 and -3 . This effect corresponds to the ability of myxothiazole to inhibit glucose-induced hyperpolarization, followed by depolarization of the $\Delta \Psi_{\mathrm{M}}$, and with inhibition of glucose generated ROS. At $24 \mathrm{~h}$, even though rapid MMD was blocked, there was a slight increase in caspase cleavage even in the presence of myxothiazole, an event associated with a reduction in glycolytic capacity.

Similar to results obtained with myxothiazole, another inhibitor of the electron, transfer chain reduces induction of PCD. TTFA inhibits Mt complex II and has uncoupling properties, although in this study TTFA did not exhibit strong uncoupling effects. TTFA blocked generation of ROS in neurons, similar to observations in endothelial cells $(13,22)$, and partially reduced glucose-induced Mt enlargement and caspase-3 cleavage, although this was less complete than with myxothiazole. Rotenone, which only partially inhibits transfer of electrons from complex I, is ineffective in blocking generation of ROS in cells exposed to high glucose (13). Overall, the data with electron transfer chain inhibitors are consistent with a model in which a hyperglycemia-induced increase in electron transfer donors from the tricarboxylic acid cycle $(\mathrm{NADH}$ and $\mathrm{FADH}_{2}$ ) causes hyperpolarization of the $\Delta \Psi_{\mathrm{M}}$ by pumping protons across the Mt inner membrane. In turn, this drives reduction of $\mathrm{O}_{2}$ to generate superoxide anions $(13,17,23)$.

The glucose-induced rise in oxidative stress is associated with translocation of cytochrome $c$ from the Mt into the cytosol, an event that precedes initiation of PCD (1). A frequent, but not invariable, requirement for translocation of cytochrome $c$ from the intermitochondrial space to the cytosol is either Mt hyperpolarization or MMD (1). Cytochrome $c$ release from the Mt is related to an intact permeability transition pore and may be released from Mt even in the presence of a functional outer Mt membrane, possibly by regulation of pore forming sites by Bax and Bcl-2 (1). In sympathetic neurons, colocalization of cytochrome $c$ within intact $\mathrm{Mt}$ is associated with punctate staining, similar to our findings in DRG neurons. When cytochrome $c$ is translocated in the cytosol, there is loss of focal Mt staining (24) using light microscopy, although colocalization with the apoptosome may occur, a structure similar in size to a ribosome. This probably accounts for the discrete staining observed in the current study using high magnification confocal microscopy.

Translocation of cytochrome $c$ is associated with formation of an apoptosome with pro-caspase- 9 and Apaf-1 (1, 25). Subsequent cleavage of pro-caspase-9 results in downstream cleavage of effector caspases such as caspase-3 or nuclear translocation, with subsequent DNA cleavage. In DRG neurons exposed to high glucose, there is a fourfold increase in cleavage of both initiator caspases such as caspase-9 and effector caspases like caspase-3. Cleavage occurs within $6 \mathrm{~h}$ after exposure to $45 \mathrm{mM}$ glucose, but follows MMD. This is consistent with activation of caspases and downstream targets by an intrinsic PCD pathway. Cleavage of both initiator and effector caspases was blocked by inhibitors of the Mt electron transfer chain.

In summary, our study demonstrates that high glucose mediates production of ROS and oxidative stress in DRG that parallels changes in Mt size and function. Mt enlarge, hyperpolarize, and depolarize; these effects are blocked by myxothiazole and TTFA, inhibitors of ROS formation. Changes in the $\Delta \Psi_{\mathrm{M}}$ correspond not only to generation of ROS, but also to partial depletion of ATP, an event that is coupled to apoptosis. Together, the increase in ROS and impaired regulation of oxidative stressors result in PCD of DRG and provides a mechanism to explain how impaired regulation of peak glucose levels leads to ROS-induced injury in diabetic neuropathy.

The authors would like to thank Ms. Judy Boldt for assistance with preparation of the manuscript. Supported in part by National Institutes of Health (NIH) NS01938, NS42056, JDRF, Office of Research Development (Medical Research Service), Department of Veterans Affairs (J.W.R.); NIH NS36778, NIH NS38849, PFUND, and JDRF (E.L.F.); NIDDK \#5P60DK-20572, MDRTC.

\section{REFERENCES}

1. Green, D. R., and Reed, J. C. (1998) Mitochondria and apoptosis. Science 281, 1309-1312

2. Russell, J. W., Sullivan, K. A., Windebank, A. J., Herrmann, D. N., and Feldman, E. L. (1999) Neurons undergo apoptosis in animal and cell culture models of diabetes. Neurobiol. Dis. 6 347-363

3. Srinivasan, S., Stevens, M. J., and Wiley, J. W. (2000) Diabetic peripheral neuropathy: Evidence for apoptosis and associated mitochondrial dysfunction. Diabetes 49, 1932-1938

4. Russell, J. W., and Feldman, E. L. (1999) Insulin-like growth factor-I prevents apoptosis in sympathetic neurons exposed to high glucose. Horm. Metab. Res. 31, 90-96

5. Kogawa, S., Yasuda, H., Terada, M., Maeda, K., and Kikkawa, R. (2000) Apoptosis and impaired axonal regeneration of sensory neurons after nerve crush in diabetic rats. NeuroReport 11 , 663-667

6. Kishi, M., Mitsui, M., Nagamatsu, M., Nickander, K. K., Schmelzer, J. D., Tanabe, J., Yao, J. K., and Low, P. A. (2001) Dorsal root ganglion pathology in chronic experimental diabetic neuropathy. J. Peripher. Neru. Syst. 6, 152-153

7. Zochodne, D. W., Verge, V. M., Cheng, C., Sun, H., and Johnston, J. (2001) Does diabetes target ganglion neurones? Progressive sensory neurone involvement in long-term experimental diabetes. Brain 124, 2319-2334

8. Kishi, M., Tanabe, J., Schmelzer, J. D., and Low, P. A. (2002) Morphometry of dorsal root ganglion in chronic experimental diabetic neuropathy. Diabetes 51, 819-824

9. Greene, D. A., Stevens, M. J., Obrosova, I., and Feldman, E. L. (1999) Glucose-induced oxidative stress and programmed cell death in diabetic neuropathy. Eur. J. Pharmacol. 375, 217-223

10. Vanden Hoek, T. L., Becker, L. B., Shao, Z., Li, C., and Schumacker, P. T. (1998) Reactive oxygen species released from mitochondria during brief hypoxia induce preconditioning in cardiomyocytes. J. Biol. Chem. 273, 18092-18098

11. Wadia, J. S., Chalmers-Redman, R. M. E., Ju, W. J. H., Carlile, G. W., Phillips, J. L., Fraser, A. D., and Tatton, W. G. (1998) Mitochondrial membrane potential and nuclear changes in apoptosis caused by serum and nerve growth factor withdrawal: time course and modification by (-)-deprenyl. J. Neurosci. 18 $932-947 \mathrm{v}$ 
12. Eskes, R., Antonsson, B., Osen-Sand, A., Montessuit, S., Richter, C., Sadoul, R., Mazzei, G., Nichols, A., and Martinou, J. C. (1998) Bax-induced cytochrome $\mathrm{C}$ release from mitochondria is independent of the permeability transition pore but highly dependent on Mg2+ ions. J. Cell Biol. 143, 217-224

13. Nishikawa, T., Edelstein, D., Du, X. L., Yamagishi, S., Matsumura, T., Kaneda, Y., Yorek, M. A., Beebe, D., Oates, P. J., Hammes, H. P., Giardino, I., and Brownlee, M. (2000) Normalizing mitochondrial superoxide production blocks three pathways of hyperglycaemic damage. Nature (London) 404, 787-790

14. Korinkova, P., and Lodin, Z. (1976) The metabolism of glucose of nerve cells cultivated under different conditions. Acta Histochem. 56, 47-65

15. Bottenstein, J. E. (1989) Serum-free culture of dissociated chick embryo sensory neurons. In A Dissection and Tissue Culture Manual of the Nervous System (Shahar, A., de Vellis, J., Vernadakis, A., and Haber, B., eds) pp. 227-229, Alan R. Liss, New York

16. Vander Heiden, M. G., Chandel, N. S., Li, X. X., Schumacker, P. T., Colombini, M., and Thompson, C. B. (2000) Outer mitochondrial membrane permeability can regulate coupled respiration and cell survival. Proc. Natl. Acad. Sci. USA 97, 4666-4671

17. Brownlee, M. (2001) Biochemistry and molecular cell biology of diabetic complications. Nature (London) 414, 813-820

18. Stevens, M. J., Obrosova, I., Cao, X., Van Huysen, C., and Greene, D. A. (2000) Effects of DL-alpha-lipoic acid on peripheral nerve conduction, blood flow, energy metabolism, and oxidative stress in experimental diabetic neuropathy. Diabetes 49, 1006-1015
19. Sasaki, H., Schmelzer, J. D., Zollman, P. J., and Low, P. A. (1997) Neuropathology and blood flow of nerve, spinal roots and dorsal root ganglia in longstanding diabetic rats. Acta Neuropathol. 93, 118-128

20. Kroemer, G., Dallaporta, B., and Resche-Rigon, M. (1998) The mitochondrial death/life regulator in apoptosis and necrosis. Annu. Rev. Physiol. 60, 619-642

21. Zamzami, N., El Hamel, C., Maisse, C., Brenner, C., MunozPinedo, C., Belzacq, A. S., Costantini, P., Vieira, H., Loeffler, M., Molle, G., and Kroemer, G. (2000) Bid acts on the permeability transition pore complex to induce apoptosis. Oncogene 19, $6342-6350$

22. Du, X. L., Edelstein, D., Rossetti, L., Fantus, I. G., Goldberg, H., Ziyadeh, F., Wu, J., and Brownlee, M. (2000) Hyperglycemiainduced mitochondrial superoxide overproduction activates the hexosamine pathway and induces plasminogen activator inhibitor-1 expression by increasing Sp1 glycosylation. Proc. Natl. Acad. Sci. USA 97, 12222-12226

23. Vincent, A. M., Brownlee, M., and Russell, J. W. (2002) Oxidative stress and programmed cell death in diabetic neuropathy. Ann. N. Y. Acad. Sci. 959, 368-383

24. Deshmukh, M., and Johnson, E. M., Jr. (1998) Evidence of a novel event during neuronal death: development of competence-to-die in response to cytoplasmic cytochrome $c$. Neuron $\mathbf{2 1}$, 695-705

25. Pan, G., O'Rourke, K., and Dixit, V. (1998) Caspase-9, Bcl-xl, and Apaf-1 form a ternary complex. J. Biol. Chem. 273, 5841-5845

Received for publication December 21, 2001. Accepted for publication July 15, 2002. 